\title{
Profile of PEGylated interferon beta in the treatment of relapsing-remitting multiple sclerosis
}

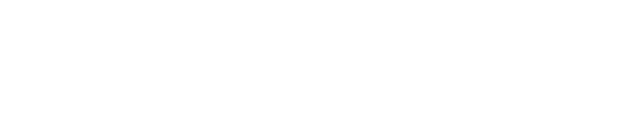

\section{Eleonora Cocco \\ Maria Giovanna Marrosu}

Multiple Sclerosis Center, Department of Public Health, Clinical and Molecular Medicine, University of Cagliari, Cagliari, Italy
Correspondence: Eleonora Cocco Multiple Sclerosis Center, Department of Public Health, Clinical and Molecular Medicine, University of Cagliari/Binaghi Hospital, Via Is Guadazzonis,

2, 09126 Cagliari, Italy

Tel +390706093027

Fax +390706092929

Email ecocco@unica.it
Abstract: Several treatments are currently available for relapsing-remitting multiple sclerosis. Among them, interferon (IFN) beta remains a valid treatment approach because of its good benefit/risk profile. Due to the need for frequent administration (weekly, at a minimum), the use of IFN beta is limited by uncomfortable side effects that could reduce adherence to and persistence with the treatment. The use of subcutaneous polyethylene glycol (PEG)ylated interferon beta-1a (PEG-IFN) has been proposed to offer a better combination of pharmacokinetic and pharmacodynamic profiles and therapy-related side effects. A $125 \mu \mathrm{g}$ dose of PEG-IFN given every 2 or 4 weeks was tested in two Phase I studies and shown to be as safe and efficient as IFN beta-1a but with a longer half-life. A Phase III trial (ADVANCE) comparing $125 \mu \mathrm{g}$ of PEG-IFN given every 2 or 4 weeks with placebo in 1,512 patients with relapsing-remitting multiple sclerosis showed significant reductions in both the annualized relapse rate (ARR) and the occurrence of new or newly enlarged T2 brain lesions in both experimental groups versus placebo after the first year. Moreover, 38\% fewer patients showed progression of disability $(P=0.04)$ in the PEG-IFN groups. During the second year, the ARR was further reduced in the PEG-IFN 2-week treatment group ( 0.230 at 1 year versus 0.178 at 2 years) and was maintained in the 4-week treatment group. Patients who received immediate PEG-IFN treatment showed improved clinical efficacy (ARR, risk of relapse, 12-week disability progression) and magnetic resonance imaging parameters (new T2 and newly enlarging lesions, gadolinium-positive lesions) compared with those with delayed treatment. The effects were more evident with the 2-week dose for all endpoints considered. Furthermore, PEG-IFN was well tolerated, and no new safety concerns arose. In conclusion, PEGIFN has good efficacy and a good safety profile. The available data support the use of PEG-IFN as a suitable therapeutic option in patients with relapsing-remitting multiple sclerosis.

Keywords: PEGylated interferon beta, multiple sclerosis, disease-modifying drug, relapses, magnetic resonance imaging, safety

\section{Interferon beta and multiple sclerosis}

Multiple sclerosis (MS) is a chronic disease of the central nervous system that can potentially cause permanent disability in young adults. ${ }^{1}$ Although the pathogenesis is unknown, MS is considered autoimmune in nature. No definitive therapies exist to cure MS, but a set of drugs is used to reduce the inflammatory activity (relapses and magnetic resonance imaging [MRI] parameters) and to delay the progression of disability over time. ${ }^{1}$

Interferon (IFN) beta has immunomodulatory properties, antiviral and antiproliferative effects, promotes cell differentiation, and exerts a pleiotropic mechanism of action in MS. ${ }^{2}$ Among other effects, IFN beta:

- Downregulates the expression of major histocompatibility complex class II molecules on various antigen-presenting cells

- Reduces the migration of T-cells across the brain barriers by decreasing the secretion of proteolytic matrix metalloproteinases and surface-expressed adhesion 
molecules and by increasing the release of soluble adhesion molecules

- Induces a shift in cytokine networks in favor of an antiinflammatory effect (Th2 cytokines)

- Suppresses T-cell proliferation

- Inhibits activation of monocytes. ${ }^{2}$

In 1993, subcutaneously administered IFN beta became the first officially approved drug available for the treatment of relapsing-remitting MS. A pivotal trial considered 372 MS patients randomized to receive placebo or IFN beta- $1 \mathrm{~b}$ at two dosages (50 or $250 \mu \mathrm{g}$ subcutaneously every other day) and showed a significantly reduced annualized relapse rate (ARR) and reduced MRI activity in patients receiving IFN beta-1b compared with placebo. Moreover, a dose-related effect was evident. ${ }^{3,4}$

After another pivotal study was completed, IFN beta-1a administered once weekly was approved in 1996. This study included $301 \mathrm{MS}$ patients with Expanded Disability Status Scale (EDSS) scores between 1.0 and 3.5 and at least two relapses in the previous 3 years. Patients were randomized to receive placebo or IFN beta-1a (30 mg intramuscularly once a week) for 2 years. ${ }^{5,6}$ The patients treated with IFN beta-1a showed significantly decreased disease activity when compared with the placebo-treated patients.,

IFN beta-1a administered subcutaneously three times weekly was investigated in the PRISMS study, ${ }^{7}$ in which 560 patients (EDSS 1.0-5.0 and at least two relapses in the preceding 2 years) were randomized to receive placebo or IFN beta-1a at two different dosages ( 22 or $44 \mu \mathrm{g}$ subcutaneously three times weekly). Treatment with IFN beta-1a significantly reduced the relapse rate and MRI activity compared with placebo treatment, with a statistically significant dose effect, and was approved for the treatment of relapsing-remitting MS in 1998 in Europe and Canada and in 2002 in the USA.

Thus, regardless of formulation, IFN beta efficiently reduced the ARR by approximately 30\% and decreased the appearance of brain MRI lesions over 2 or 3 years in patients with relapsing-remitting $\mathrm{MS} .{ }^{8} \mathrm{~A}$ dose-response effect was demonstrated in head-to-head trials (EVIDENCE and INCOMIN) comparing different IFN beta formulations, with higher doses and more frequent administration showing greater effects than lower doses. ${ }^{9,10}$

IFN beta also had a significant effect in the early stages of the disease, as demonstrated in three clinical trials (CHAMPS, ETOMS, and BENEFIT) performed in patients with clinically isolated syndrome. ${ }^{11-13}$ In these trials, early IFN beta treatment delayed the development of clinically definite MS (by $40 \%-50 \%$ ) and had a favorable effect on clinical and MRI disease activity. ${ }^{11-13}$

IFN beta was also tested for secondary progressive MS in four randomized, placebo-controlled trials, but few effects on disability progression were evident. ${ }^{14-18}$ Patients with secondary progressive MS and superimposed relapses still have a response to IFN beta- $1 b$, which is the only IFN beta approved for both relapsing-remitting and secondary progressive MS. ${ }^{14}$

IFN beta has a good safety profile, with no major adverse events, and is generally well tolerated. The most frequent side effects are injection site reactions and flu-like symptoms that decrease in intensity over time in the majority of patients. Blood alterations such as lymphopenia could occur with IFN beta treatment, and elevated liver enzymes have been reported. ${ }^{8}$ Moreover, thyroid dysfunction and some cases of thrombotic microangiopathy have been reported in association with the use of IFN beta. ${ }^{19,20}$

However, the need for frequent (weekly, at a minimum) administration of the drug (depending on the specific type) could be inconvenient and uncomfortable for some patients and could reduce their adherence to and persistence with treatment.

A recent review systematically evaluated the tolerability of and adherence to MS therapies, analyzing data from both randomized controlled trials and observational studies. ${ }^{21}$ For IFN beta, the most common adverse events were flulike symptoms and injection site reactions, and the mean discontinuation rates ranged from $16 \%$ to $27 \%$. Although no differences in the tolerability or adherence data from randomized controlled trials and observational studies were observed, the frequency of side effects continued to be high in long-term studies, and drug suspensions accumulated over time. ${ }^{21}$

\section{PEGylation}

One way to decrease the dose frequency while maintaining efficacy is to conjugate IFN beta with polyethylene glycol (PEG). PEGylation of IFN is done by covalently joining a PEG molecule to an IFN molecule. PEGylation has been applied to several drugs for different diseases to increase drug stability, solubility, half-life, and efficacy. ${ }^{22}$

If the protein is small, PEGylation is optimal because it reduces the renal clearance and improves the half-life of the protein. PEGylation can cause loss of biological activity in large, unstable proteins. Thus, PEGylation is not recommended in every case. ${ }^{23}$ Although infrequent, anti-PEG antibodies have been observed. When repeated chronic doses of PEGylated 
proteins are given, anti-PEG antibodies may potentially increase the clearance of proteins attached to PEG. ${ }^{24}$

Currently, more than ten PEGylated products are marketed, including pegylated interferon (PEG-IFN) alpha-2a and alpha- $2 b$ for the treatment of hepatitis $C$ and certolizumab pegol with the parent drug anti-TNF Fab for the treatment of rheumatoid arthritis and Crohn's disease. Further, PEGylated IFN beta-1a was tested with or without ribavirin in hepatitis $\mathrm{C}$, yielding improvements in liver biopsies. ${ }^{25}$

\section{Studies of PEG-IFN in animals}

IFN beta contains a relatively small, 166 amino acid, singlechain $22.5 \mathrm{kDa}$ glycoprotein with a half-life of 10 hours. Thus, to optimize IFN therapy in MS, PEGylation is a suitable method. A set of over 20 site-selective mono-PEGylated or multi-PEGylated IFN beta-1b bioconjugates were studied by Basu et $\mathrm{al}^{26}$ in mice and rats with regard to stability, solubility, aggregation, immunogenicity, and in vivo exposure of recombinant human IFN beta-1b. Selected PEGylated IFN beta- 1 b compounds showed a reduced humoral response with decreased immunoglobulin $\mathrm{G}$ and an expanded area under the curve (AUC) exposure with respect to IFN beta-1b.

Another study conducted by Mager et al evaluated the subcutaneous administration of $4 \mathrm{kDa}$ PEG-IFN beta-1a in monkeys and revealed increased exposure and pharmacological activity similar to that of standard IFN beta- $1 \mathrm{a} .{ }^{27}$

\section{PEG-IFN preparations for MS in development}

Several PEG-IFN beta preparations are in development, ${ }^{28}$ including ARX-424 (Merck Serono with Ambrx Inc) and the recombinant human IFN beta BaroFeron ${ }^{\mathrm{TM}}$ (BaroFold Inc). The latter showed acceptable safety and tolerability profiles in a recent Phase I trial. ${ }^{25}$ PEG-IFN beta-1b NU400, derived from aggregate-free IFN NU100 (identical to BaroFeron), recently entered a Phase I trial. A subcutaneously administered site-specific PEGylated form of human IFN beta-1b (AZ01, Allozyne Inc) is under evaluation in a multiple ascending dose Phase IB trial for the treatment of relapsingremitting MS. ${ }^{28}$ Preliminary results suggest that the drug was well tolerated, while causing the well-known side effects of IFN beta. ${ }^{28}$

\section{PEG-IFN beta- I a}

Phase III studies of PEG-IFN beta-1a (developed by Biogen Idec) were recently concluded, and the drug received positive recommendations in the summer of 2014 as a treatment for relapsing-remitting MS by the International authorities in the USA and Europe (US Food and Drug Administration and European Medicines Agency, respectively).

PEG-IFN beta-1a is obtained by adding a $20 \mathrm{kDa}$ methoxyPEG-O-2-methylpropionaldehyde to the alpha amino group of the IFN beta-1a N-terminus. ${ }^{29}$ In vitro PEG-IFN beta-1a testing showed approximately 50\% activity in antiviral and antiproliferative assays. However, its activity in vivo (mouse model) was enhanced when compared with IFN beta-1a. Moreover, the pharmacokinetics and biological activity of PEG-IFN beta-1a were improved in animal models (rats and rhesus monkeys). ${ }^{29}$

PEG-IFN beta-1a was then studied in two randomized, blinded Phase I studies ${ }^{29}$ in healthy volunteers: a single-dose study comparing IFN beta-1a (30 $\mu$ g intramuscularly) with PEG-IFN beta-1a at different dosages $(63,125$, or $188 \mu \mathrm{g}$ intramuscularly or subcutaneously) in 60 individuals; $;^{29}$ and a multiple-dose study comparing PEG-IFN beta-1a administered subcutaneously once every 2 or 4 weeks versus placebo in 69 individuals. ${ }^{29}$ In these studies, the pharmacokinetic and pharmacodynamic profiles and immune activity, safety, and tolerability measures were assessed.

PEG-IFN beta-1a exposure increased proportionally to the dose; the exposure of PEG-IFN beta-1a was fourfold greater at $63 \mu \mathrm{g}(6 \mathrm{MIU})$ than that of IFN beta-1a at $30 \mu \mathrm{g}$ (6 MIU). ${ }^{29}$ Immunological effects, including neopterin and $2^{\prime}, 5^{\prime}$-OAS levels and changes in T-helper cell pathway gene expression and lymphocyte subsets, were dose-dependent and larger and more persistent with PEG-IFN beta-1a than with IFN beta- $1 \mathrm{a} .^{29}$

The peak pharmacological effect was at 12 hours for PEG-IFN beta-1a versus 6 hours for IFN beta-1a. Additionally, the activity of PEG-IFN beta-1, measured by a specific enzyme-linked immunosorbent assay and a cell-based antiviral cytopathic effect bioassay, was detectable in the blood for $\leq 7$ days compared with 2 days for IFN beta-1a. Further, the response was sustained for up to 15 days for PEG-IFN beta- $1 \mathrm{a}$ and 4 days for IFN beta-1a. ${ }^{29}$

In both studies, PEG-IFN beta-1a had a good safety and tolerability profile; no serious adverse events occurred, and no patients discontinued their trial medication because of adverse events. No differences in adverse events were observed between PEG-IFN (88\%-100\%) and INF beta (92\%). ${ }^{29}$

The most frequent side effect was the typical flu-like syndrome, which appears to be dose-dependent but decreases after recurrent dosing. Notably, the flu-like syndrome persisted after repeated dosing with $188 \mu \mathrm{g}$ PEG-IFN beta-1a given every 4 weeks. A more frequent transient reduction in absolute number of neutrophils $\left(>1,500\right.$ cells $\left./ \mathrm{mm}^{3}\right)$ was 
observed with PEG-IFN administered every 2 weeks versus 4 weeks, as well as with IFN beta. The neutropenia generally resolved 2 weeks after drug administration. Elevated liver enzymes were observed in both studies, but the levels were generally below the threefold upper limit, with a mean increase of 1.2-1.3.

Using a cell-based neutralizing antibody assay to detect binding or neutralizing IFN beta antibodies, the serum samples from subjects included in the studies tested negative. ${ }^{29}$

Prior to the first dose, six subjects were found to be anti-PEG antibody-positive, and no increases in titer were observed during the study. Five individuals developed antiPEG antibodies during the study, but considering their rarity, their effect was unclear.

In the single-dose study, no differences between subcutaneous and intramuscular dose administration were observed. Considering that subcutaneous administration is better tolerated and considered more convenient by patients, this route was chosen for the Phase III study.

The $125 \mu \mathrm{g}$ PEG-IFN dose yielded 50\% less exposure than the $188 \mu \mathrm{g}$ dose, but biological marker (neopterin) levels were only slightly lower. This dose was better tolerated compared with the $188 \mu \mathrm{g}$ dose in terms of severity of flu-like symptoms, injection site reactions, and blood test abnormalities. Therefore, the $125 \mu \mathrm{g}$ dose was chosen for the Phase III clinical study. Compared with the standard IFN beta-1a dose (30 $\mu \mathrm{g}$ intramuscularly) administered once weekly, the subcutaneously administered $125 \mu \mathrm{g}$ PEG-IFN beta-1a showed a 5.4-fold higher AUC when administered every 2 weeks and a 2.7-fold higher AUC when administered every 4 weeks. $^{29}$

The degrees of renal impairment in the pharmacokinetics of PEG-IFN after a single dose $(63 \mu \mathrm{g}$ or $125 \mu \mathrm{g})$ were studied, with the results showing that the AUC and peak plasma concentration were similar for healthy subjects and patients with end-stage renal disease receiving hemodialysis. Thus, dose adjustment in subjects with renal impairment is not mandatory. ${ }^{30}$

\section{Phase III ADVANCE study}

The first clinical trial to evaluate the efficacy of PEG-IFN beta-1a in relapsing-remitting MS is ADVANCE study (Efficacy and Safety Study of BIIB017), ${ }^{31}$ which is registered with ClinicalTrials.gov (NCT00906399). ${ }^{31}$ ADVANCE is a randomized, placebo-controlled, double-blind (for the first year) and dose-blind (for the second year), parallel-group, multicenter study with 183 sites in 26 countries.

At the beginning of the study, 1,516 patients with relapsing-remitting MS were randomized (via an interactive voice response or web system and stratified by site) into three treatment arms $(1: 1: 1)$ as follows: PEG-IFN beta-1a at a dose of $125 \mu \mathrm{g}$ every 2 weeks (PEG-IFN 2w), PEGIFN beta-1a at a dose of $125 \mu \mathrm{g}$ every 4 weeks (PEG-IFN $4 \mathrm{w}$ ) and placebo. Prefilled syringes were provided to the patients for the duration of the study. The inclusion criteria were: age 18-65 years, relapsing-remitting MS, an EDSS score of $\leq 5.0$, and at least two relapses during the previous 3 years, with at least one in the preceding year. The exclusion criteria comprised secondary progressive MS, presence of laboratory abnormalities, previous treatment with IFN beta for MS for more than 4 weeks, or discontinuation of IFN beta after less than 6 months.

Patients who were initially randomized into the placebo group were rerandomized into one of the treatment arms of the study at the end of the first year (48 weeks) to receive either the low (4 weeks) or high (2 weeks) frequency PEGIFN treatment. ${ }^{32}$

The primary endpoint of the study was the ARR at 1 year (48 weeks). Secondary outcomes were the total number of new or newly enlarging MRI T2 lesions, the proportion of patients with relapses, and progression of disability (variation of the EDSS) at 48 weeks. Tertiary endpoints were the number of gadolinium-enhancing lesions, new T1 hypointense lesions, new active lesions (gadolinium lesions plus new or enlarging T2 lesions), volumes of new enlarging lesions, T1 gadolinium-positive lesions, T1 hypointense lesions, brain atrophy, and the magnetization transfer ratio. ${ }^{31}$

Of the 1,936 patients originally screened for ADVANCE, 1,512 received at least one dose of the study medication. Of these, 512 were randomized to PEG-IFN 2w, 500 to PEGIFN 4w, and 500 to placebo. In total, 88\% (1,332 patients) completed the first 48 weeks of the study. ${ }^{31}$

The adjusted ARRs were 0.397 (95\% confidence interval [CI] 0.328-0.481) in the placebo arm, 0.256 (95\% CI 0.206-0.318) in the PEG-IFN 2w arm, and 0.288 (95\% CI $0.234-0.355)$ in the PEG-IFN $4 \mathrm{w}$ arm, with a reduction in ARR compared with the placebo arm of $35.6 \%(P<0.001)$ and $27.5 \%$ $(P<0.02)$, respectively, in the PEG-IFN $2 \mathrm{w}$ and $4 \mathrm{w}$ arms. ${ }^{31}$

The risk of 12-week confirmed sustained disability progression was reduced by $38 \%(P<0.04)$ in both active treatment arms versus the placebo arm. The proportion of patients who experienced a relapse was reduced by $39 \%$ $(P<0.001)$ in the PEG-IFN $2 \mathrm{w}$ arm and 26\% $(P<0.03)$ in the PEG-IFN 4w arm.

The number of new or newly enlarged T2 lesions observed by brain MRI was reduced by $67 \%(P<0.001)$ in the PEG-IFN $2 \mathrm{w}$ arm and 28\% $(P<0.001)$ in the PEG-IFN $4 \mathrm{w}$ arm at 48 weeks. ${ }^{31}$ PEG-IFN $2 \mathrm{w}$ reduced the number 
Table I Primary and secondary endpoints of the ADVANCE trial

\begin{tabular}{|c|c|c|c|c|c|c|}
\hline & \multicolumn{3}{|l|}{ First year } & \multicolumn{3}{|l|}{ Second year } \\
\hline & $\begin{array}{l}\text { PEG-IFN } \\
2 \text { weeks }\end{array}$ & $\begin{array}{l}\text { PEG-IFN } \\
4 \text { weeks }\end{array}$ & Placebo & $\begin{array}{l}\text { PEG-IFN } \\
2 \text { weeks }\end{array}$ & $\begin{array}{l}\text { PEG-IFN } \\
4 \text { weeks }\end{array}$ & $\begin{array}{l}\text { Delayed } \\
\text { PEG-IFN }\end{array}$ \\
\hline Total number & 512 & 500 & 500 & 512 & 500 & 500 \\
\hline Annualized relapse rate & 0.256 & 0.288 & 0.397 & 0.221 & 0.291 & 0.35 \\
\hline$(95 \% \mathrm{Cl})$ & $(0.206-0.318)$ & $(0.234-0.355)$ & $(0.328-0.48 I)$ & $(0.183-0.267)$ & $(0.244-0.348)$ & $(0.295-0.418)$ \\
\hline \multicolumn{7}{|l|}{ Proportion of patients } \\
\hline \multicolumn{7}{|l|}{ with a relapse } \\
\hline Number & 90 & 105 & 142 & 124 & 158 & 192 \\
\hline Proportion & 0.187 & 0.222 & 0.291 & 0.265 & 0.344 & 0.402 \\
\hline \multicolumn{7}{|l|}{ Disability progression } \\
\hline \multicolumn{7}{|l|}{ (12 weeks confirmed) } \\
\hline Number & 31 & 31 & 50 & 51 & 56 & 75 \\
\hline Proportion & 0.068 & 0.068 & 0.105 & 0.112 & 0.123 & 0.162 \\
\hline \multicolumn{7}{|l|}{ New or newly enlarging } \\
\hline \multicolumn{7}{|l|}{ T2 lesion } \\
\hline $\begin{array}{l}\text { Number of patients } \\
\text { evaluated }\end{array}$ & 457 & 462 & 476 & 407 & 389 & 393 \\
\hline $\begin{array}{l}\text { Adjusted mean } \\
\text { lesion number }\end{array}$ & 3.6 & 7.9 & 10.9 & 5.0 & 12.5 & 14.8 \\
\hline
\end{tabular}

Notes: Results for the first ${ }^{31}$ and second year ${ }^{32}$ are presented. Reprinted from Lancet Neurol, I3, Calabresi PA, Kieseier BC, Arnold DL, et al. ADVANCE Study Investigators. Pegylated interferon $\beta$-la for relapsing-remitting multiple sclerosis (ADVANCE): a randomised, Phase 3, double-blind study. 657-665., Copyright (20I4), with permission from Elsevier. ${ }^{31}$ Reprinted from Kieseier BC, Arnold DL, Balcer LJ, et al. Peginterferon beta-la in multiple sclerosis: 2-year results from ADVANCE. Mult Scler. Copyright ${ }^{\odot}$ 2014. Reprinted by Permission of SAGE. ${ }^{32}$

Abbreviations: $\mathrm{Cl}$, confidence interval; PEG-IFN, polyethylene glycol (PEG)ylated interferon.

of new or newly enlarged T2 hyperintense lesions versus both placebo and PEG-IFN $4 \mathrm{w}$ at both $24(61 \%$ and $51 \%$, respectively, $P<0.0001)$ and 48 (67\% and 54\%, respectively, $P<0.0001)$ weeks. ${ }^{33}$ Results on the primary and secondary endpoints are summarized in the table 1. PEG-IFN $2 \mathrm{w}$ also significantly reduced the number of new $\mathrm{T} 1$ hypointense, gadolinium-positive lesions and new active (gadoliniumpositive plus non-enhancing new T2) lesions (all $P<0.0001$ ), as well as the volumes of T2 and T1 lesions $(P<0.05)$ at 24 and 48 weeks versus placebo and PEG-IFN 4w. ${ }^{33}$

A post hoc analysis was then performed to evaluate the effect of PEG-IFN when assessing for "no evidence of disease activity", defined as the absence of clinical (any relapses and confirmed disability progression at 12 weeks) or MRI (new or newly enlarging T2 hyperintense and gadolinium-positive lesions) disease activity. ${ }^{33}$ Significantly more patients in the PEG-IFN 2w arm had no evidence of disease activity versus the placebo and PEG-IFN 4w arms (all $P<0.01$ ) from baseline to week 24 (41.0\% versus $21.9 \%$ and $30.7 \%$; odds ratio 2.47 and 1.57$)$, from week 24 to week $48(60.2 \%$ versus $28.9 \%$ and $36.6 \%$; odds ratio 3.71 and 2.62 ) and from baseline to week 48 (33.9\% versus $15.1 \%$ and $21.5 \%$; odds ratio 2.89 and 1.87 ). ${ }^{33}$

Adverse events were reported by 417 (83\%) patients taking placebo, 481 (94\%) taking PEG-IFN 2w, and 472 (94\%) taking PEG-IFN 4w. ${ }^{31}$ The most common adverse events accompanying PEG-IFN were injection site reactions, flu-like symptoms, pyrexia, and headaches. Flu-like symptoms were the most common adverse event leading to treatment discontinuation in the PEG-IFN groups. The most common serious adverse events were relapse and infections ( $\leq 1 \%$ in each treatment group) ${ }^{31}$ their frequencies were similar in both PEG-IFN groups. Specifically, these adverse events were present in $53(11 \%)$ patients taking placebo, $90(18 \%)$ patients taking PEG-IFN 2w, and $82(16 \%)$ patients taking PEG-IFN 4w. Reduced hematological parameters and increased liver enzymes were more commonly observed in the active treatment arms than in the placebo arm, but no drug discontinuation occurred due to blood alterations. ${ }^{31}$ Neutralizing antibodies against IFN beta-1a developed in a small proportion of patients and were generally transient. ${ }^{31}$

\section{Results for the second year of ADVANCE}

At the end of the first year (48 weeks), patients randomized to placebo were rerandomized into one of the active treatment arms (228 in each group). Patients already included in one of the PEG-IFN arms continued treatment at the same dosing regimen for the rest of the study (438 in each group). The discontinuation rate due to adverse events was similar between dosing regimens ( $6 \%$ in each arm). ${ }^{32}$

The ARR was further reduced in the second year in the PEG-IFN $2 \mathrm{w}$ arm $(0.230$ at 1 year and 0.178 at 2 years $)$ and was maintained in the PEG-IFN 4w arm (0.286 at 1 year and 0.291 at 2 years). The number of new or newly 
enlarged lesions was lower in the second year versus the first year in both the PEG-IFN 2w (1.9 versus 4.1) and PEG-IFN 4w (5.6 versus 9.4) arms. ${ }^{32}$

Comparing patients who started the active treatment at the beginning of the study (continuous PEG-IFN) in the first year with those who started PEG-IFN later (delayed PEGIFN), the greater efficacy of early treatment was evident. ${ }^{32}$ In particular, the ARR was 0.351 for delayed treatment, 0.021 for PEG-IFN 2w, and 0.291 for PEG-IFN 4w. The post hoc analysis showed an ARR reduction of $37 \%(P<0.0001)$ for PEG-IFN 2w and of 17\% ( $P=0.0906)$ for PEG-IFN $4 \mathrm{w}$ versus delayed treatment. ${ }^{32}$ The proportion of patients who relapsed in the 2 years was 0.42 for delayed treatment and 0.265 and 0.344 for PEG-IFN $2 \mathrm{w}$ and $4 \mathrm{w}$, respectively. Thus, the risk of relapse was reduced by $39 \%(P<0.0001)$ in the PEG-IFN $2 \mathrm{w}$ arm and $19 \%(P=0.0465)$ in the PEG-IFN $4 \mathrm{w}$ arm. ${ }^{32}$

The 12-week disability progression was reduced by $33 \%$ $(P=0.0257)$ in the PEG-IFN $2 \mathrm{w}$ arm and $25 \%(P=0.0960)$ in the PEG-IFN 4w arm. At 24 weeks, disability progression was reduced by $41 \%(P=0.0137)$ for PEG-IFN $2 \mathrm{w}$ and by $9 \%(P=0.6243)$ for PEG-IFN 4w. ${ }^{32}$ Moreover, patients treated with continuous versus delayed PEG-IFN presented fewer new T2 or newly enlarged lesions after 2 years (reduction of $67 \%$ and $16 \%$ for PEG-IFN $2 \mathrm{w}$ and $4 \mathrm{w}$, respectively). The number of gadolinium-positive lesions was also reduced with continuous PEG-IFN 2w compared with delayed PEG-IFN 2w (0.2 versus $0.5 ; P=0.0002){ }^{32}$

Comparison of the PEG-IFN 2w and PEG-IFN 4w dose regimens revealed a better effect of PEG-IFN $2 w$. In particular, the ARR was 0.221 in the PEG-IFN 2w arm and 0.29 in the PEG-IFN 4w arm, with the advantage of a $24 \%$ reduction for PEG-IFN $2 \mathrm{w}$ versus the PEG-IFN 4w. ${ }^{32}$ Additionally, the risk of relapse was reduced by $24 \%(P=0.0212)$ in the PEG-IFN 2w arm when compared to the PEG-IFN 4w arm. Hazard ratios indicated that PEG-IFN $2 \mathrm{w}$ reduced the risk of confirmed disability progression at 12 weeks by $11 \%$ $(P=0.5665)$ and at 24 weeks by $36 \%(P=0.0459)$ compared with PEG-IFN 4w. ${ }^{32}$ Over 2 years, the PEG-IFN $2 \mathrm{w}$ arm had $60 \%(P<0.0001)$ fewer new or newly enlarged hyperintense T2 lesions and a reduced number of gadolinium-positive lesions by approximately $71 \%(P<0.0001)$ compared with the PEG-IFN 4w arm. ${ }^{32}$

Results on the primary and secondary endpoints are summarized in the Table 1. The incidence of adverse events over 2 years was similar (approximately 94\%) between the PEGIFN treatment groups. The most commonly reported events were injection site reactions, flu-like symptoms, pyrexia, and headaches. ${ }^{32}$ The adverse events were generally mild or moderate, and the incidence of severe adverse events was similar between the two PEG-IFN dosing groups (21\% and $20 \%$, respectively, for PEG-IFN $2 \mathrm{w}$ and $4 \mathrm{w}$ ). The incidence of adverse events during the second year was similar to that in the first year. ${ }^{32}$ Nine deaths were reported over the 2-year study period, but none were considered to be related to PEG-IFN. In the second year, less than $10 \%$ of patients presented significant abnormalities in white blood cell counts, lymphocyte counts, and absolute neutrophil counts. The majority of hepatic transaminase elevations were less than three times the upper limit of normal. Moreover, for both dosing regimens, abnormal values for hepatic or hematological laboratory parameters returned to normal at the end of year $2 .^{32}$ The incidence of anti-IFN neutralizing antibodies was $<1 \%$ in both the PEG-IFN 2w and 4w arms, and antibodies against PEG-IFN were present in $8 \%$ and $6 \%$ of patients in the PEG-IFN 2w and 4w arms, respectively. ${ }^{32}$

\section{Extension study (ATTAIN)}

At the end of the core study (2 years), patients were invited to enter into the extension trial, ie, ATTAIN (Long-Term Safety and Efficacy Study of BIIB017), a 2-year-long, open-label, dose frequency blinded study. Data are not yet available because the study is ongoing. A substudy evaluated a singleuse autoinjector for PEG-IFN versus prefilled syringes in a small group of 39 patients, and showed that patients preferred the autoinjector for its convenience. ${ }^{34}$

\section{A warning from animal studies and other PEG formulations}

In Sprague-Dawley rats (male and female), daily intravenous doses of PEG-linked tumor necrosis factor-binding protein for 3 months caused vacuolation of the renal cortical tubular epithelium. ${ }^{35}$ The kidney lesions only partially recovered after 2 months, in the absence of changes in kidney function tests. Similar lesions were observed with other PEG-linked proteins of similar weight. ${ }^{36}$ The majority of tubular vacuolation occurred in animals exposed to doses several times higher than those administered in clinical practice..$^{35,36}$

\section{Discussion and conclusion}

The therapeutic landscape for MS is rapidly changing, and several new drugs have reached the market or will likely reach the market in the near future. This situation is exciting for MS specialists because, to date, the response to available drugs has been suboptimal due to the heterogeneity of the disease. An enriched armament will bring new opportunities for individualized treatment but will also generate 
new challenges regarding efficacy and safety issues in the long term, and detailed discussions with patients about the risk/benefit balance before treatment decisions will become more and more important. Some drugs, such as fingolimod, natalizumab, and mitoxantrone, considered as second-line therapies, potentially offer greater efficacy but are burdened by an increased risk of serious adverse events. While the first-line therapies, ie, IFN beta and glatiramer acetate, have well-established efficacy and good long-term safety profiles, they come with disturbing and uncomfortable side effects in addition to the need for frequent administration via an unpleasant route. Considering that these aspects could lead MS patients to reduce their adherence to and persistence with treatment, optimization of treatment with IFN beta by PEGylation has recently been proposed. PEGylation of IFN beta molecules improves the pharmacodynamic and pharmacokinetic profile by increasing the half-life while also maintaining efficacy. The addition of a PEG molecule to IFN beta increases the size of the molecule, thereby increasing the persistence of the drug in the circulation by decreasing the rate of kidney clearance or reducing proteolysis and opsonization and leading to an increased half-life and drug efficacy. ${ }^{29}$

Recently, PEG-IFN beta-1a, administered by two injections every 2 weeks, has been recommended for use in patients with relapsing-remitting MS by international authorities, including the US Food and Drug Administration in the USA and the European Medicines Agency in Europe. PEGIFN beta- $1 \mathrm{a}$ at a dose of $125 \mu \mathrm{g}$ taken every 2 or 4 weeks was tested in two Phase I studies in healthy subjects and shown to be at least as safe and effective as the current IFN beta-1a administered intramuscularly. ${ }^{29}$

The effect of treating relapsing-remitting MS patients with $125 \mu \mathrm{g}$ PEG-IFN every 2 or 4 weeks (subcutaneously) was tested in the Phase III ADVANCE study, showing in the first year a reduction of the ARR by approximately one-third compared with placebo, and slight but significant reductions in sustained disability progression and in several MRI activity measures were also shown. ${ }^{31-33}$

The efficacy data ${ }^{31-33}$ seem to be similar to those observed in the pivotal trials of IFN beta, ${ }^{3-7}$ suggesting a similar effect of PEG-IFN. However, it should be kept in mind that "head to head" or "not inferiority" trials comparing PEG-IFN with IFN beta directly have not been performed yet. Moreover, the comparison of trials performed in different decades could be misleading because the clinical and diagnostic characteristics of the MS patients included in the studies have changed over time. ${ }^{37,38}$
Additionally, a post hoc analysis performed during the first 48 weeks of ADVANCE showed that significantly more patients with relapsing-remitting MS receiving PEG-IFN every 2 weeks had no evidence of disease activity. ${ }^{33}$ Further, results from the second year of ADVANCE showed a greater reduction in all endpoints with dosing every 2 weeks versus every 4 weeks. The effects of PEG-IFN taken every 2 weeks appeared to increase in the second year; this was a surprising observation in contrast to the effects of IFN beta. ${ }^{32}$

The drug was well tolerated, with the most common adverse events being flu-like symptoms, redness, injection site reactions, and headache. The rate of serious adverse events was low, with a frequency of severe infection of less than $1 \%{ }^{28,29}$ No cases of thyroid dysfunction or thrombotic microangiopathy were observed in ADVANCE. ${ }^{31-33}$ The safety and tolerability profile of PEG-IFN seems to be similar to that of IFN beta, but it is noteworthy that no data from Phase IV trials or observational studies or long-term information are actually available. Notably, the chronic administration of toxic concentrations of PEGylated proteins caused vacuolation of the renal epithelium in animals..$^{35,36}$ No evidence of such damage was observed with PEG-IFN beta-1a in humans, but a careful monitoring program is advisable. ${ }^{31-33}$

In conclusion, due to its frequency of administration, along with its good efficacy and safety profiles, PEG-IFN beta-1a represents a suitable therapeutic option for patients with relapsing-remitting MS.

\section{Disclosure}

The authors report no conflicts of interest in this work.

\section{References}

1. Kamm CP, Uitdehaag BM, Polman CH. Multiple sclerosis: current knowledge and future outlook. Eur Neurol. 2014;72:132-141.

2. Kasper LH, Reder AT. Immunomodulatory activity of interferon-beta. Ann Clin Transl Neurol. 2014;1:622-631.

3. [No authors listed]. Interferon $b-1 b$ is effective in relapsing-remitting multiple sclerosis. I. Clinical results of a multicenter, randomized, double-blind, placebo-controlled trial. The IFNB Multiple Sclerosis Study Group. Neurology. 1993;43:655-661.

4. Paty DW, Li DK. Interferon beta $\mathrm{lb}$ is effective in relapsing-remitting multiple sclerosis. II. MRI analysis results of a multicenter, randomized, double-blind, placebo-controlled trial. Neurology. 1993;43:662-667.

5. Rudick RA, Goodkin DE, Jacobs LD, et al. Impact of interferon beta-1a on neurologic disability in relapsing multiple sclerosis. The Multiple Sclerosis Collaborative Research Group (MSCRG). Neurology. 1997;49:358-363

6. Simon JH, Jacobs LD, Campion M, et al. Magnetic resonance studies of intramuscular interferon beta-1a for relapsing multiple sclerosis. The Multiple Sclerosis Collaborative Research Group. Ann Neurol. 1998;43:79-87.

7. [No authors listed]. Randomised double-blind placebo controlled study of interferon beta-1a in relapsing/remitting multiple sclerosis. PRISMS (Prevention of Relapses Disability by Interferon beta-1a Subcutaneously in Multiple Sclerosis) Study Group. Lancet. 1998;352:1498-1504. 
8. Annibali V, Mechelli R, Romano S, et al. IFN- $\beta$ and multiple sclerosis: from etiology to therapy and back. Cytokine Growth Factor Rev. October 31, 2014. [Epub ahead of print].

9. Panitch H, Goodin DS, Francis G, et al. Randomized, comparative study of interferon b-1a treatment regimens in MS. The EVIDENCE Trial. Neurology. 2002;59:1496-1506.

10. Durelli L, Verdun E, Barbero P, et al. Every-other-day interferon beta-1b versus once-weekly interferon beta-1a for multiple sclerosis: results of a 2-year prospective randomized multicentre study (INCOMIN). Lancet. 2002;359:1453-1460.

11. Jacobs LD, Beck RW, Simon JH, et al. Intramuscular interferon-beta-1a therapy initiated during a first demyelinating event in multiple sclerosis. N Engl J Med. 2000;343:898-904.

12. Comi G, Filippi M, BarkhofF, et al. Effect of early interferon treatment on conversion to definite multiple sclerosis: a randomized study. Lancet. 2001;357:1576-1582.

13. Kappos L, Polman CH, Freedman MS, et al. Treatment with interferon beta-1b delays conversion to clinically definite and McDonald MS in patients with clinically isolated syndromes. Neurology. 2006;67: $1242-1249$.

14. [No authors listed]. Placebo-controlled multicentre randomised trial of interferon beta-1b in treatment of secondary progressive MS. European Study Group on Interferon Beta-1b in Secondary Progressive MS. Lancet. 1998;352:1491-1497.

15. Panitch H, Miller A, Paty D, Weinshenker B; North American Study Group on Interferon beta-1b in Secondary Progressive MS. Interferon beta-1b in secondary progressive MS: results from a 3-year controlled study. Neurology. 2004;63:1788-1795.

16. Secondary Progressive Efficacy Clinical Trial of Recombinant Interferonbeta-1 in MS (SPECTRIMS) Study Group. Randomized controlled trial of interferon-beta-1a in secondary progressive MS: clinical results. Neurology. 2001;56:1496-1504.

17. Li DK, Zhao GJ, Paty DW; University of British Columbia MS/MRI Analysis Research Group, The SPECTRIMS Study Group. Randomized controlled trial of interferon-beta-1a in secondary progressive MS: MRI results. Neurology. 2001;56:1505-1513.

18. Cohen JA, Cutter GR, Fischer JS, et al. Benefit of interferon beta-1a on MSFC progression in secondary progressive MS. Neurology. 2002;59: 679-687.

19. Frisullo G, Calabrese M, Tortorella C, et al. Thyroid autoimmunity and dysfunction in multiple sclerosis patients during long-term treatment with interferon beta or glatiramer acetate: an Italian multicenter study. Mult Scler. 2014;20:1265-1268.

20. Hunt D, Kavanagh D, Drummond I, et al. Thrombotic microangiopathy associated with interferon beta. N Engl J Med. 2014;370: 1270-1271.

21. Giovannoni G, Southam E, Waubant E. Systematic review of diseasemodifying therapies to assess unmet needs in multiple sclerosis: tolerability and adherence. Mult Scler. 2012;18:932-946.

22. Bailon P, Won CY. PEG-modified biopharmaceuticals. Expert Opin Drug Deliv. 2009;6:1-16.
23. Pisal DS, Kosloski MP, Balu-Iyer SV. Delivery of therapeutic proteins. J Pharm Sci. 2010;99:2557-2575.

24. Wang $X$, Ishida T, Kiwada H. Anti-PEG IgM elicited by injection of liposomes is involved in the enhanced blood clearance of a subsequent dose of PEGylated liposomes. J Control Release. 2007;119: 236-244.

25. Rao HY, Li J, Zhang LF, et al. [Effect of PEGylated interferon beta-1a therapy on the liver fibrosis in chronic hepatitis $\mathrm{C}$ : a semi-quantitative analysis]. Zhonghua Yi Xue Za Zhi. 2008;88:96-100. Chinese.

26. Basu A, Yang K, Wang M, et al. Structure-function engineering of interferon beta-1b for improving stability, solubility, potency, immunogenicity, and pharmacokinetic properties by site-selective monoPEGylation. Bioconjug Chem. 2006;17:618-630.

27. Mager DE, Neuteboom B, Jusko WJ. Pharmacokinetics and pharmacodynamics of pegylated IFN-beta 1a following subcutaneous administration in monkeys. Pharm Res. 2005;22:58-61.

28. Reuss R. PEGylated interferon beta-1a in the treatment of multiple sclerosis - an update. Biologics. 2013;7:131-138.

29. Hu X, Miller L, Richman S, et al. A novel PEGylated interferon beta-1a for multiple sclerosis: safety, pharmacology, and biology. J Clin Pharmacol. 2012;52:798-808.

30. $\mathrm{Hu} \mathrm{X}$, Seddighzadeh A, Stecher S, et al. Pharmacokinetics, pharmacodynamics, and safety of peginterferon beta-1a in subjects with normal or impaired renal function. J Clin Pharmacol. 2015;55:179-188.

31. Calabresi PA, Kieseier BC, Arnold DL, et al; ADVANCE Study Investigators. Pegylated interferon $\beta$-1a for relapsing-remitting multiple sclerosis (ADVANCE): a randomised, Phase 3, double-blind study. Lancet Neurol. 2014;13:657-665.

32. Kieseier BC, Arnold DL, Balcer LJ, et al. Peginterferon beta-1a in multiple sclerosis: 2-year results from ADVANCE. Mult Scler. November 28, 2014. [Epub ahead of print].

33. Arnold DL, Calabresi PA, Kieseier BC, et al. Effect of peginterferon beta-1a on MRI measures and achieving no evidence of disease activity: results from a randomized controlled trial in relapsing-remitting multiple sclerosis. BMC Neurol. 2014;14:1058.

34. Seddighzadeh A, Hung S, Selmaj K, et al. Single-use autoinjector for peginterferon- $\beta 1$ a treatment of relapsing-remitting multiple sclerosis: safety, tolerability and patient evaluation data from the Phase IIIb ATTAIN study. Expert Opin Drug Deliv. 2014;11:1713-1720.

35. Bendele A, Seely J, Richey C, Sennello G, Shopp G. Short communication: renal tubular vacuolation in animals treated with polyethyleneglycol-conjugated proteins. Toxicol Sci. 1998;42:152-157.

36. Young MA, Malavalli A, Winslow N, Vandegriff KD, Winslow RM. Toxicity and hemodynamic effects after single dose administration of MalPEG-hemoglobin (MP4) in rhesus monkeys. Transl Res. 2007;149: 333-342.

37. Inusah $\mathrm{S}$, Sormani MP, Cofield SS, et al. Assessing changes in relapse rates in multiple sclerosis. Mult Scler. 2010;16:1414-1421.

38. Sormani MP. The Will Rogers phenomenon: the effect of different diagnostic criteria. J Neurol Sci. 2009;287:S46-S49.
Therapeutics and Clinical Risk Management

\section{Publish your work in this journal}

Therapeutics and Clinical Risk Management is an international, peerreviewed journal of clinical therapeutics and risk management, focusing on concise rapid reporting of clinical studies in all therapeutic areas, outcomes, safety, and programs for the effective, safe, and sustained use of medicines. This journal is indexed on PubMed Central, CAS,

\section{Dovepress}

EMBase, Scopus and the Elsevier Bibliographic databases. The manuscript management system is completely online and includes a very quick and fair peer-review system, which is all easy to use. Visit $\mathrm{http}: / /$ www.dovepress.com/testimonials.php to read real quotes from published authors. 